\title{
Estrutura populacional de Aegla schmitti (Crustacea: Anomura: Aeglidae) nos reservatórios dos Mananciais da Serra, Piraquara, Paraná, Brasil
}

\author{
Érico A. O. Teodósio \& Setuko Masunari
}

\author{
Departamento de Zoologia, Universidade Federal do Paraná. Caixa Postal 19020, 81531-980 Curitiba, Paraná, Brasil. \\ E-mail: setmas@ufpr.br
}

\begin{abstract}
Population structure of Aegla schmitti (Crustacea: Anomura: Aeglidae) in the reservoirs of Mananciais da Serra, Piraquara city, state of Paraná, Brazil. A study on population structure of Aegla schmitti Hobbs III, 1979 was carried out at Mananciais da Serra, in the municipality of Piraquara, state of Paraná, Brazil $\left(25^{\circ} 29^{\prime} \mathrm{S}, 4^{\circ} 58^{\prime} \mathrm{W}\right)$. The following aspects were treated: temporal fluctuation in abundance, sex ratio, reproductive period and size composition based on carapace length. The animals were colleted with traps, nets, measured and returned to the reservoirs. This population was sampled monthly, in two reservoirs (Carvalho and Carvalhinho) constructed in the headwater streams with swift current and steep gradient, located on west side of Serra do Mar mountains, from November, 2004 to January, 2006. The water temperature varied from 11.0 (July, 2005) to $18.2^{\circ} \mathrm{C}$ (March, 2007), dissolved oxygen from 7.0 to $9.5 \mathrm{mg} / \mathrm{l}$ and $\mathrm{pH}$ from 4.8 to 6.7 , during collection period. A total of 1230 individuals were collected, among which, 819 were males and 411 females (30 ovigerous). The abundance varied from 23 (December, 2004) to 122 (June, 2005) crabs and the sex ratio of the whole population was 1:0.5 (M:F). Male carapace length varied from 4.38 to $36.46 \mathrm{~mm}$ and female, from 4.28 to $32.56 \mathrm{~mm}$. Ovigerous females occurred from April, 2005 to November, 2005. Higher crab abundance was recorded in the Carvalho Reservoir, which explained by the presence of numerous breaches on the old tank walls that acted as shelters for these animals. Aegla schmitti females incubate egg mass during colder months and its juveniles hatch mainly in spring, as most Aegla Leach, 1820 species occurring in southeastern and southern Brazil.
\end{abstract}

KEY WORDS. Aeglid crab; sex ratio; temporal distribution.

Os crustáceos anomuros de água doce atuais estão agrupados em um único gênero, Aegla Leach, 1820, com cerca de 61 espécies ou subespécies endêmicas à região Neotropical. Dessas espécies, 35 ocorrem no Brasil (Bond-Buckup \& Buckup 1994). Aegla spp. são habitantes de arroios, riachos, rios, lagos e cavernas e são encontrados em profundidades variadas, desde águas rasas de riachos até $320 \mathrm{~m}$ de profundidade em lagos chilenos e, em altitudes de até $4500 \mathrm{~m}$ acima do nível do mar, em rios da Cordilheira dos Andes (Bond-Buckup 2003).

Há diversos estudos populacionais sobre eglas no Brasil. No Estado de São Paulo, López (1965) estudou a biologia de Aegla odebrechtii paulensis Schmitt, 1942 (hoje A. paulensis Schmitt, 1942) nos rios do Alto da Serra, Paranapiacaba e Rodrigues \& Hebling (1978) estudaram A. perobae Hebling \& Rodrigues, 1977 em riachos de São Pedro. Mais recentemente, Fransozo et al. (2003) estudaram uma população de $A$. castro Schmitt, 1942 no Córrego Itaúna, Itatinga. No Estado do Rio Grande do Sul, aspectos populacionais foram desenvolvidos por diversos autores, dentre eles: Bueno \& Bond-Buckup (2000) com A. platensis Schmitt, 1942 nos arroios de Taquara; Noro \& Buckup (2002), com A. leptodactyla Buckup \& Rossi, 1977 no Rio da Divisa, São José dos Ausentes; Colpo et al. (2005) com A. longirostri Bond-Buckup \& Buckup,
1994 proveniente do Rio Ibicuí-Mirim, Itaára; GonÇALves et al. (2006) com A. franciscana Buckup \& Rossi, 1977 do Rio Rolante, São Francisco de Paula. No Estado do Paraná, apenas os trabalhos de Swiech-Ayoub \& Masunari (2001a, b) que trataram de aspectos populacionais e reprodutivos de $A$. castro ocorrente no Buraco do Padre, Ponta Grossa, estão disponíveis.

A rápida degradação da qualidade das águas nos ambientes límnicos da América do Sul exige aceleração dos estudos sobre as biotas aquáticas continentais. Os eglídeos são vulneráveis às mudanças em seus habitats, e há sérios riscos destes animais entrarem em extinção local antes mesmo de serem conhecidos (BOND-Buckup \& Buckup 1994).

Aegla schmitti Hobbs III, 1979 tem distribuição geográfica restrita ao Brasil e sua ocorrência abrange o sudeste do Estado de São Paulo, nordeste, leste e sudeste do Paraná e norte de Santa Catarina (Bond-Buckup \& Buckup 1994). Apesar de sua ampla distribuição geográfica, a literatura não contempla qualquer aspecto populacional desta espécie. O presente artigo tem como objetivo descrever a estrutura populacional de $A$. schmitti, com base na distribuição temporal, proporção de sexos, aspectos da biologia reprodutiva e composição de tamanho da população amostrada. 


\section{MATERIAL E MÉTODOS}

O trabalho foi desenvolvido nos Mananciais da Serra $\left(25^{\circ} 29^{\prime} \mathrm{S}, 48^{\circ} 58^{\prime} \mathrm{W}\right)$ que estão localizados no município de Piraquara, região metropolitana de Curitiba, distando cerca de $40 \mathrm{~km}$ da capital. São constituídos por um conjunto de rios e riachos que pertencem à Bacia do Alto Iguaçu, numa região com características de Floresta Ombrófila Densa ou Floresta Atlântica e de mata ciliar bem preservada. A área possui 2249 ha e está situada em altitudes de 900 a 1450 m, junto à vertente oeste da Serra do Mar (Vicentini et al. 1991). Pelo sistema de Köeppen, a região apresenta clima subtropical úmido, mesotérmico, sem estação seca, com verões frescos e geadas freqüentes no inverno $(\mathrm{Cfb})$.

No período de estudo, a temperatura média mensal do ar variou de $13,3^{\circ} \mathrm{C}$ (julho/2005) a $21,3^{\circ} \mathrm{C}$ (janeiro/2006), e a precipitação de 46,2 mm (dezembro/2005) a 210,0 mm (setembro/2005) na região dos Manancias da Serra (SimEPAR 2006).

O Reservatório Carvalho tem formato semi-circular com capacidade de $800 \mathrm{~m}^{3}$ e está localizado numa área aberta, fora da mata. Ele é provido ainda de seis tanques de concreto, que acomodaram filtros de areia no passado (hoje retirados). Estes tanques têm aproximadamente seis metros de profundidade e estão interconectados por meio de aberturas localizadas a cerca de quatro metros do fundo, nível em que se encontra a superfície da água de modo contínuo. As águas captadas em outros reservatórios menores têm passagem obrigatória através destes compartimentos antes de serem represadas no reservatório propriamente dito. Folhas mortas e matéria orgânica em decomposição estão sempre presentes no fundo de todos os tanques de filtros.

O Reservatório Carvalhinho possui apenas uma barragem de concreto como forma de retenção de água, com profundidade de dois metros e capacidade de aproximadamente $200 \mathrm{~m}^{3}$. Está localizado numa área permanentemente sombreada, dentro da mata, seu fundo é coberto com areia e folhas mortas, juntamente com cascalho fino e grosso e fragmentos de galhos de árvore. Os dois reservatórios distam $80 \mathrm{~m}$ um do outro.

Espécimens de A. schmitti foram coletados mensalmente, nos dois reservatórios descritos acima, no período de novembro/2004 a janeiro/2006, totalizando 15 coletas. Os animais foram coletados com o auxílio de armadilhas do tipo covo, as quais foram montadas com isca de ração úmida para gatos, e armadas dentro dos compartimentos de filtros. Redes do tipo "hand net" com $2 \mathrm{~mm}$ de abertura de malha foram utilizadas para capturar os poucos animais que escalavam as paredes dos tanques. Os animais foram recolhidos das armadilhas e o comprimento da carapaça (CC - ponta do rostro até o bordo posterior do cefalotórax) medido comum paquímetro digital Mitutoyo, com 0,01 mm de precisão.

O sexo de cada espécime foi determinado in loco, pela presença de pleópodos nas fêmeas e ausência nos machos. Os indivíduos menores do que $8 \mathrm{~mm}$ de $\mathrm{CC}$, foram coletados, transportados ao laboratório, onde o sexo foi determinado pela ob- servação da presença dos poros genitais femininos nas coxas do terceiro par de pereiópodos das fêmeas. Esses exemplares foram conservados em álcool a 70\%. A presença de fêmeas ovígeras foi anotada no campo, para determinar o período reprodutivo da espécie. Os animais com CC inferior ao da menor fêmea ovígera coletada, foram tratados como juvenis (imaturos sexualmente), os demais foram considerados adultos. Para verificar a possível ocorrência de diferenças significativas na proporção de machos e fêmeas e de animais entre os reservatórios, ao longo do período amostral, foi utilizado o teste $\chi^{2}$ (quiquadrado). Após a obtenção dos parâmetros biológicos acima citados, in loco, os espécimes maiores que $8 \mathrm{~mm}$ de CC foram liberados no hábitat de origem.

O esforço de coleta foi padronizado inspecionadas as armadilhas a cada 30 minutos, durante cinco horas. No Reservatório Carvalho, foram utilizadas 10 armadilhas e no Reservatório Carvalhinho, quatro. Em cada coleta foram tomados, também, os seguintes dados abióticos em cada reservatório: temperatura do ar, com um termômetro de mercúrio comum; temperatura e oxigênio dissolvido na água, com um oxigenômetro de campo Hanna Instruments, modelo HI 9145; pH com um potenciômetro de campo Ingolf, modelo PH-206, e observação visual das condições climáticas (com sol, nublado, chuva) por ocasião da coleta. As temperaturas e precipitações médias mensais foram obtidas junto ao Simepar (Sistema Meteorológico do Paraná), na Estação Meteorológica de Piraquara. Afim de comparar os dados abióticos de cada reservatório, acima descritos, foi utilizado o teste "t" de Student, com nível de significância de 5\%.

Os parâmetros populacionais foram analisados considerando os caranguejos obtidos nos dois reservatórios como uma única população. Os animais foram distribuídos em classes de CC com intervalo de $2 \mathrm{~mm}$, o que corresponde a um quarto do desvio padrão da média calculada para o somatório de animais analisados (MARKus 1971), totalizando 17 classes. Estas foram denominadas tanto no texto como nos gráficos com os respectivos pontos médios.

Para facilitar a visualização da estrutura populacional em termos de CC ao longo do período de estudo, os animais foram agrupados trimestralmente como segue: primero trimestre fevereiro, março e abril/2005, segundo. trimestre - maio, junho e julho/2005, terceiro trimestre - agosto, setembro e outubro/2005 e quarto trimestre - novembro, dezembro/2005 e janeiro/2006. As coletas dos meses de novembro, dezembro/2004 e janeiro/2005 não foram incluídas nessa análise para que o período compreendesse um ano.

\section{RESULTADOS}

No Reservatório Carvalho, a temperatura do ar variou de 12,0 (julho/2005) a $26,5^{\circ} \mathrm{C}$ (outubro/2005) e a temperatura da água, de 10,8 (julho/2005) a $17,9^{\circ} \mathrm{C}$ (outubro/2005). O valor mínimo do oxigênio dissolvido foi de $6,2 \mathrm{mg} / \mathrm{l}$ (janeiro/2006) e o máximo de 9,2 mg/l (julho/2005). O pH variou de 4,7 (outubro/2005) a 6,7 (novembro/2004). No Reservatório 
Carvalhinho, a temperatura mínima do ar ocorreu em julho/ $2005\left(11^{\circ} \mathrm{C}\right)$ e a maior em janeiro/2006 $\left(21,0^{\circ} \mathrm{C}\right)$. A temperatura da água variou de 11,0 (julho/2005) e $18,2^{\circ} \mathrm{C}$ (março/2005). $\mathrm{O}$ menor valor de oxigênio dissolvido foi de $7 \mathrm{mg} / \mathrm{l}$ (janeiro/ 2006) e o maior de $9,5 \mathrm{mg} / \mathrm{l}$ (julho/2005). O pH variou de 4,8 (julho/2005) a 6,7 (novembro/2004). Não houve diferença significativa entre os dois reservatórios quanto às variáveis abióticas analisadas ao longo do período de estudo.

As condições climáticas por ocasião das coletas apresentaram-se com sol em doze dos quinze meses de coleta e nublado com chuvisco em três meses.

Foram obtidos 1230 indivíduos de $A$. schmitti ao longo do período de estudo, sendo 819 machos e, 411 fêmeas (30 ovígeras), perfazendo respectivamente 67 e 33\% da população. No Reservatório Carvalho, foram coletados 970 indivíduos (706 machos, 264 fêmeas, das quais, 29 ovígeras), enquanto no Carvalhinho, 260 exemplares (113 machos, 147 fêmeas, das quais, uma ovígera).

A abundância de $A$. schmitti variou de 23 (dezembro/2004) a 122 (junho/2005), com dois picos na flutuação de abundância: um em fevereiro/2005, com 107 indivíduos e outro em junho/2005, com 122 (Fig. 1).

A proporção de sexos foi de 1:0,5 (M:F) quando analisa-

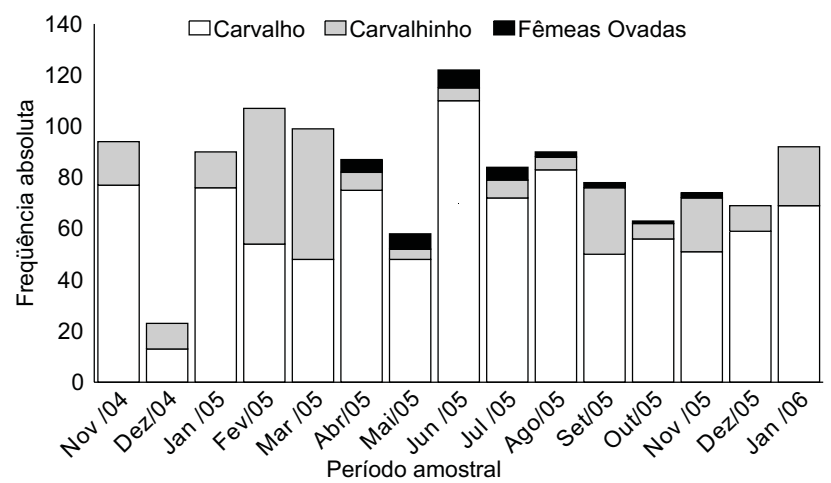

Figura 1. Variação anual da abundância na população de A. schmitti, ao longo do período amostral nos Mananciais da Serra.

da a população como um todo. Entretanto, ela foi significativamente diferente no Carvalho $-\chi^{2}(3,84)=100,7 ; p<0,05$, onde houve predominância de machos, enquanto no Carvalhinho não houve diferença significativa $-\chi^{2}(3,84)=2,22$; $\mathrm{p}<$ 0,05; neste último reservatório, o número total de fêmeas foi um pouco superior ao dos machos.

As fêmeas ovígeras perfizeram $7 \%$ do total de fêmeas coletadas e apenas $2 \%$ da população. Estas foram obtidas de abril/2005 a novembro/2005, com maior freqüência (50\%) em maio/2005, seguido de abril/2005 e julho/05 (ambos com 24\%).

A amplitude de variação do CC nos machos foi de 4,38 a $36,46 \mathrm{~mm}$ e nas fêmeas, de 4,28 a $32,56 \mathrm{~mm}$, os quais foram distribuídos em 17 e 15 classes de CC, respectivamente (Fig. 2).
A distribuição de freqüência de animais amostrados nas classes de CC apresentou-se bimodal para ambos os sexos. Dentre os machos, a primeira classe modal de CC foi a de $9,0 \mathrm{~mm}$ e a segunda, a de $27,0 \mathrm{~mm}$ e, para as fêmeas, a de 13,0 e a de 21,0 $\mathrm{mm}$, respectivamente. Os machos atingiram maiores CC do que as fêmeas, visto que estas não ocuparam as duas últimas classes; além disso, elas apresentaram um número menor de indivíduos nas classes 31,0 e 33,0 mm (dois indivíduos e um, respectivamente) (Figs 2 e 3 ).
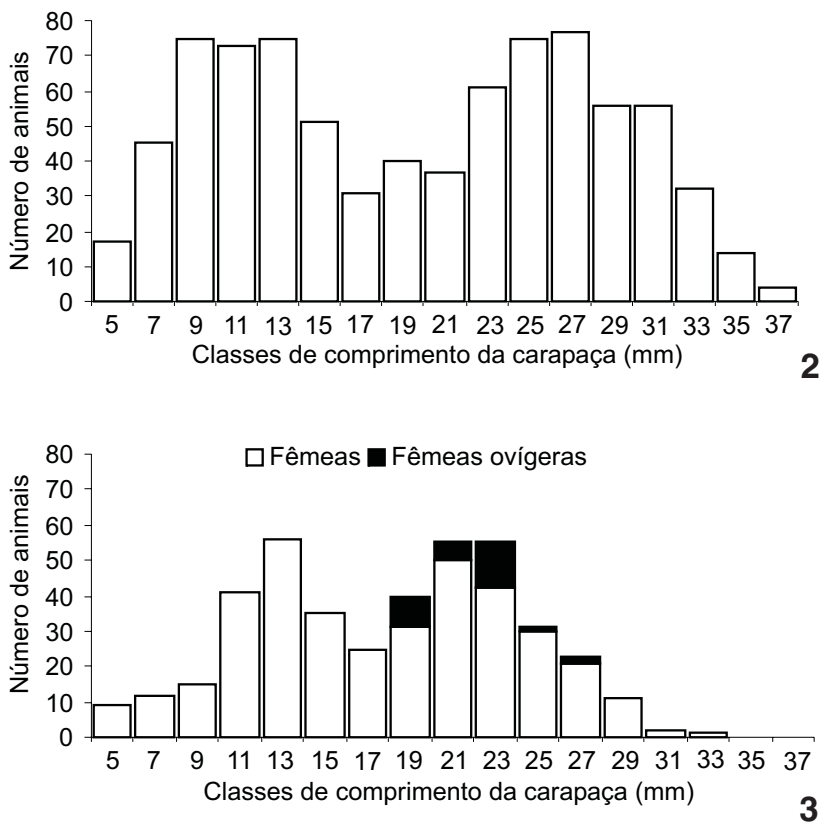

Figuras 2-3. Distribuição de freqüência de tamanho de machos (2) e fêmeas (3) de A. schmitti nas classes de comprimento da carapaça (CC).

O tamanho das fêmeas ovígeras variou de 18,10 a 27,12 $\mathrm{mm}$ de CC e foram distribuídas nas classes 19,0, 21,0, 23,0, 25,0 e 27,0 mm, com maior freqüência na 23,0 mm seguida de 19,0 mm. Os ovos aderidos aos pleópodos das fêmeas são arredondados e a coloração variou do vermelho até o laranja claro. Quanto mais adiantado o estágio de desenvolvimento embrionário, mais clara a cor do ovo em função do gradativo consumo de vitelo por parte do embrião.

A distribuição de machos e fêmeas em classes de CC nos trimestres do período estudado mostrou que os machos mantiveram a bimodalidade na maioria dos trimestres.

Dentre os machos, as classes de CC estiveram representadas integralmente (de 5,0 a 37,0 mm) em todos os trimestres (exceção do segundo trimestre na classe 37,0 mm). Somente no quarto trimestre, a referida bimodalidade não foi aparente. As fêmeas mostraram uma distribuição inconsistente numericamente, com algumas classes sem representantes (Figs 4 e 5). 

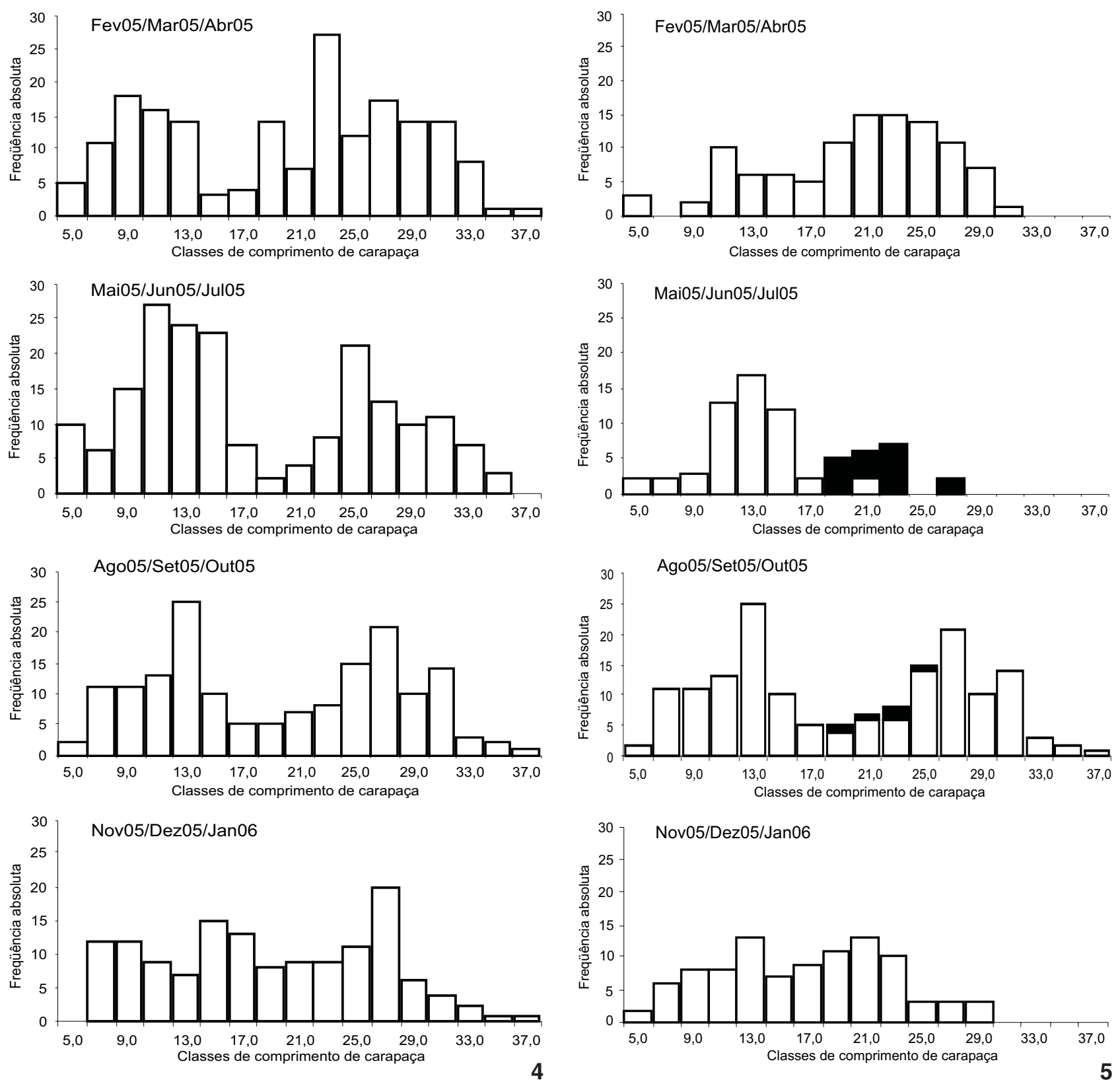

Figuras 4-5. Distribuição da freqüência absoluta de machos (4) e fêmeas (5) de A. schmitti nas classes de comprimento da carapaça (CC). As áreas escuras representam número de fêmeas ovígeras.

\section{DISCUSSÃO}

Temperaturas baixas da água e altos teores de oxigênio dissolvido parecem constituir uma condição determinante para a presença de eglídeos em ambientes de água continental, como observado neste trabalho e por BAHAMONDE \& López (1961) com Aegla laevis laevis (Latreille, 1818) no Rio Maipo, El Monte, Chile, por López (1965) e Rodrigues \& Hebling (1978) com espécies de São
Paulo; Bond-Buckup \& Buckup (1994), Bueno et al. (2000), Noro \& Buckup (2002) e Colpo et al. (2005) com eglídeos do Rio Grande do Sul e Swiech-Ayoub \& Masunari (2001b), com A. castro no Paraná.

Embora as características abióticas das águas do Reservatório Carvalho e Carvalhinho não apresentassem diferenças estatisticamente significativas (possivelmente da comunicação por meio de dutos de escoamento de água entre os dois reservatórios), a abundância de caranguejos no Carvalho foi muito 
superior. Numa primeira análise, esta diferença pode estar relacionada com a disponibilidade diferenciada de microhabitats nos dois reservatórios. O Carvalho, em função da existência de numerosas reentrâncias que formavam verdadeiras galerias no interior das paredes espessas e envelhecidas dos tanques para filtros, as quais funcionaram como abrigos perfeitos contra luz solar direta e contra correntezas muito fortes, permitiram o estabelecimento de A. schmitti neste local. Em contraste, no Carvalhinho, como descrito anteriormente, o fundo é forrado por uma espessa camada de areia, cobrindo praticamente toda altura das paredes e, portanto, diminuindo a complexidade estrutural do hábitat. O comportamento de fototaxia negativa e/ou de hábito noturno em eglídeos observados por BAHAMONDE \& López (1961) explica a necessidade de se abrigarem em refúgios e, consequentemente, foi o fator determinante da abundância de A. schmitti no Reservatório Carvalho.

Diferentemente da grande maioria dos trabalhos que abordaram a proporção de sexos em eglídeos, $A$. schmitti apresentou predomínio de machos na população dos Mananciais da Serra, um resultado que foi obtido somente por López (1965) para $A$. odebrechtii paulensis, por Rodrigues \& Hebling (1978) para $A$. perobae e por Noro \& Buckup (2002) para A. leptodactyla. Mortalidade e comportamento de modo diferenciado para machos e fêmeas podem interferir ou determinar o predomínio de um dos sexos (BAhamonde \& López 1961, Swiech-Ayoub \& Masunari 2001b).

A diferença na proporção de sexos observada nos dois reservatórios está provavelmente relacionada com o hábito diferenciado dos dois sexos. Em laboratório, foi observado que as fêmeas principalmente ovígeras, têm uma tendência a se enterrar em substrato inconsolidado no fundo do aquário. Por outro lado, os machos quase sempre ficavam expostos e com frequência nadavam na coluna d'água. Portanto, os machos parecem mais suscetíveis à captura com puçá ou atraídos pela isca das armadilhas.

Como observado em Swiech-Ayoub \& Masunari (2001a) para $A$. castro Schmitt, o registro de poucas fêmeas ovígeras durante o período de estudo, pode estar relacionado com estratégias de reprodução, fazendo com que elas procurem habitats mais protegidos, dificultando a sua amostragem. Entretanto, a descontinuidade do período reprodutivo parece caracterizar as espécies de Aegla ocorrentes na região sul e sudeste do Brasil: das dez espécies cujo período reprodutivo é conhecido, sete têm esta característica (Tab. I). Além disso, os períodos de incubação dos ovos de oito espécies, juntamente com A. schmitti, coincidem integralmente, ocorrendo no período do inverno (junho a setembro), com poucas espécies antecedendo ou estendendo em alguns meses. A população de A. castro de Itatinga, São Paulo estudada por Fransozo et al. (2003), tem pico de abundância de fêmeas ovígeras em maio e junho, mas, o período de incubação dos ovos pode ser mais extenso como ocorre com a população desta mesma espécie em Ponta Grossa, Paraná (Swiech-Ayoub \& Masunari 2001a).

Tabela I. Períodos reprodutivos das diversas espécies de Aegla distribuídas em ordem crescente de latitude sul das respectivas áreas de estudo. A extensão das barras representa os meses de duração deste período. O destaque escuro indica os meses coincidentes de ocorrência de fêmeas ovígeras.

\begin{tabular}{|c|c|c|c|c|c|c|c|c|c|c|c|c|c|}
\hline Espécies & Latitude Sul & Jan & $\mathrm{Fev}$ & Mar & Abr & Mai & Jun & Jul & Ago & Set & Out & Nov & Dez \\
\hline A. perobae ${ }^{1}$ & $22^{\circ} 33^{\prime}$ & & & & & & & & & & & & \\
\hline A. castro (Itatinga-SP) ${ }^{2}$ & $23^{\circ} 08^{\prime}$ & & & & & & & & & & & & \\
\hline A. paulensis ${ }^{3}$ & $23^{\circ} 74^{\prime}$ & & & & & & & & & & & & \\
\hline A. castro (Ponta Grossa-PR) ${ }^{4}$ & $25^{\circ} 05^{\prime}$ & & & & & & & & & & & & \\
\hline A. schmitti ${ }^{5}$ & $25^{\circ} 29^{\prime}$ & & & & & & & & & & & & \\
\hline A. leptodactyla ${ }^{6}$ & $28^{\circ} 38^{\prime}$ & & & & & & & & & & & & \\
\hline A. longirostri ${ }^{7}$ & $29^{\circ} 24^{\prime}$ & & & & & & & & & & & & \\
\hline A. franciscana $^{8}$ & $29^{\circ} 26^{\prime}$ & & & & & & & & & & & & \\
\hline A. platensis ${ }^{9}$ & $29^{\circ} 46^{\prime}$ & & & & & & & & & & & & \\
\hline A. laevis laevis ${ }^{10}$ & $33^{\circ} 44^{\prime}$ & & & & & & & & & & & & \\
\hline
\end{tabular}


O pico de abundância de fêmeas ovígeras destas espécies de Aegla nos meses com baixas temperaturas e que antecedem o início da primavera parece estar atrelado a uma vantagem na eclosão dos juvenis nos meses da primavera, quando a temperatura tende a subir até meados do verão. Certamente, em condições ambientais favoráveis como maior disponibilidade de alimento nos meses mais quentes, o desenvolvimento dos juvenis é incrementado antes da chegada dos meses mais frios. No início do inverno, estes juvenis já teriam uma idade de 6-7 meses e com desenvolvimento suficiente para enfrentar águas de baixa temperatura. Como observado por Rodrigues \& Hebling (1978), em geral, a postura dos ovos em Aegla spp. deve ocorrer no outono, a incubação no inverno e a eclosão dos juvenis na primavera. Os dados da tabela I confirmam esta predição para a maioria das espécies, independentemente da latitude de ocorrência das mesmas, uma vez que, baixas temperaturas poderiam limitar a duração da época de reprodução e influenciar o período de ocorrência no ano.

Em estudos com A. franciscana, Gonçalves et al. (2006) observaram que o tamanho dos juvenis amostrados no campo era relativamente maior do que os juvenis recém-eclodidos; isto permitiu inferir que seria possível que aqueles juvenis fossem oriundos de um processo de recrutamento ocorrido nas estações anteriores. Com o mesmo raciocínio, pode-se dizer que, no presente estudo, o ingresso de juvenis na população (classe $5,0 \mathrm{~mm}$ ) foi evidente nos primeiro e segundo trimestres, ou seja, seis ou sete meses após o pico de freqüência de fêmeas ovígeras (maio a julho) (Fig. 3), o que certamente constitui um indício para a estimativa da época de recrutamento dos juvenis na população de $A$. schmitti.

\section{AGRADECIMENTOS}

A CAPES pela concessão de bolsa de mestrado para o primeiro autor. Ao Simepar pelos dados climatológicos. À Ana C. do R. Barros e Márcia M. de Lima, Diretoria do Meio Ambiente e Ação Social da Sanepar pela autorização na execução do presente projeto nos Mananciais da Serra. A Ubiratã de A.T. da Silva, pelas fotos subaquáticas do micro-hábitat de A. schmitti. Aos coordenadores do Programa de Pós-graduação em Zoologia, UFPR, pelas facilidades oferecidas durante o desenvolvimento do projeto. À Mariângela Di Benedetto pelo auxílio nas coletas do material biológico e obtenção dos dados morfométricos. Esta é a contribuição número 1680 do Departamento de Zoologia, Universidade Federal do Paraná.

\section{LITERATURA CITADA}

Bahamonde, N. \& M.T. López. 1961. Estudios biológicos en la población de Aegla laevis laevis (Latreille) de el Monte (Crustacea, Decapoda, Anomura). Investigaciones Zoológicas Chilenas 7: 19-58.

Bond-Buckup, G. \& L. Buckup. 1994. A família Aeglidae (Crustacea, Decapoda, Anomura). Arquivos de Zoologia 32 (4): 159-346.
Bond-Buckup, G. 2003. Família Aeglidae, p. 21-116. In: G.A.S. Melo (Ed.). Manual de identificação dos crustacea decapoda de água doce do Brasil. São Paulo, Loyola, 429p.

Bueno, A.A.P. \& G. Bond-Buckup. 2000. Dinâmica populacional de Aegla platensis Schmitt (Crustacea, Decapoda, Aeglidae). Revista Brasileira de Zoologia 17 (1): 43-49.

Bueno, A.A.P.; G. Bond-Buckup \& L. Buckup. 2000. Crescimento de Aegla platensis Schmitt em ambiente natural (Crustacea, Decapoda, Aeglidae). Revista Brasileira de Zoologia 17 (1): 51-60.

Colpo, K.D.; L.O. Ribeiro \& S. Santos. 2005. Population biology of the freshwater anomuran Aegla longirostri (Aeglidae) from south Brazilian streams. Journal of Crustacean Biology 25 (3): 495-499.

Fransozo, A.; R. C. Costa; A.L.D. Reigada \& J.M. Nakagaki. 2003. Population structure of Aegla castro Schmitt, 1942 (Crustacea: Anomura: Aeglidae) from Itatinga (SP), Brazil. Acta Limnologica Brasiliensia 15 (2): 13-20.

Gonçalves, R.S.; D.S. Castiglioni \& G. Bond-Buckup. 2006. Estrutura populacional de Aegla franciscana (Crustacea, Decapoda, Anomura) em São Francisco de Paula, RS, Brasil. Iheringia, Série Zoologia, 96 (1): 109-114.

López, M.T. 1965. Estudios biológicos en Aegla odebrechtii paulensis Schmitt. Boletim de Zoologia Faculdade de Filosofia, Ciências e Letras 25: 301-314.

Markus, R. 1971. Elementos de estatística aplicada da Faculdade de Agronomia e Veterinária da UFRGS. Porto Alegre, Centro Acadêmico Leopoldo Cortez, 329p.

Noro, C.K. \& L. Buckup. 2002. Biologia reprodutiva e ecologia de Aegla leptodactyla Buckup \& Rossi (Crustacea, Anomura, Aeglidae). Revista Brasileira de Zoologia, 19 (4): 1063-1074.

Rodrigues, W. \& N. Hebling. 1978. Estudos biológicos em Aegla perobae Hebling \& Rodrigues, 1977 (Decapoda, Anomura). Revista Brasileira de Biologia 38 (2): 383-390.

SIMEPAR. 2006. Tecnologia e Informações Ambientais. Curitiba, Sistema Meteorológico do Paraná. Available online at: http:/ /www.simepar.br [Accessed: 06/II/2006].

Swiech-Ayoub, B.P. \& S. Masunari. 2001a. Biologia reprodutiva de Aegla castro Schmitt, 1942 (Crustacea, Anomura, Aeglidae) no Buraco do Padre, Ponta Grossa, Paraná, Brasil. Revista Brasileira de Zoologia 18 (3): 1019-1030.

Swiech-Ayoub, B.P. \& S. Masunari. 2001b. Flutuação temporal e espacial de abundância e composição de tamanho de Aegla castro Schmitt, 1942 (Crustacea, Anomura, Aeglidae) no Buraco do Padre, Ponta Grossa, Paraná, Brasil. Revista Brasileira de Zoologia 18 (3): 1003-1017.

Vicentini, A.; E.P. Kearney \& F. Bonato. 1991. Proposta de plano de manejo - Parque Estadual dos Mananciais da Serra. Curitiba, Departamento de Silvicultura e Manejo, Universidade Federal do Paraná, 71p.

Submitted: 22.III.2008; Accepted: 15.XII.2008.

Editorial responsibility: Neusa Hamada 